\title{
Epidemiología de la infección humana por hantavirus en Chile
}

\author{
E.U. VIVIANA SOTOMAYOR P. ${ }^{1}$ y XIMENA AGUILERA S. ${ }^{1}$
}

\section{EPIDEMIOLOGY OF HANTAVIRUS INFECTION IN CHILE}

Hantavirus Pulmonary Syndrome caused by (HPS) is an emerging disease in Chile, 123 cases of HPS were verified from 1993 to July 2000, including 5 retrospectively diagnosed cases. This disease has especially affected young people (mean 26 years old), most of them were men (75\%). The infection threatens persons living in rural communities, during farm and forest or domestic activities. The global lethality has been $49.6 \%$, with variation from $60 \%$ in 1997 to $43 \%$ in the year 2000. Cases have been reported from the sixth to elevenh regions, the highest risk has been observed in Aysén (1997-1998) and Araucanía (1998 and 2000), being prominent the appearance of clusters in the Araucanía, Bío Bío, Los Lagos and Aysén regions. Hantavirus Pulmonary Syndrome is an endemic disease with increased incidence during winter and summer time.

Key words: Hantavirus Pulmonary Syndrome; Chilean epidemiology; National Surveillance System.

\section{INTRODUCCION}

Las enfermedades causadas por hantavirus surgieron en Chile como tema emergente a mediados de la década del noventa ${ }^{1}$; sin embargo, investigaciones posteriores hacen suponer que la enfermedad existía anteriormente en nuestro país.

La característica de Hantavirus como género es su asociación con roedores específicos, que son sus hospederos y reservorios naturales. Los roedores infectados eliminan el virus por la saliva, deposiciones y orina. La forma de transmisión es la inhalación de aerosoles provenientes de orina u saliva o por el contacto directo con excretas de roedores infectados ${ }^{2}$.

Clínicamente se han descrito dos síndromes provocados por hantavirus: la fiebre hemorrágica con síndrome renal (FHSR) y el síndrome pulmonar por hantavirus (SPH), que sólo ha sido notificado en el continente americano ${ }^{3}$. En América, el SPH se describió por primera vez en E.U.A. en 1993, a raíz de un brote de una enfermedad respiratoria de elevada letalidad, que permitió identificar un nuevo hantavirus ${ }^{4}$, al que se denominó Virus Sin Nombre (VSN) ${ }^{5,6}$. La enfermedad se caracterizó con un período prodrómico de corta duración, con fiebre, mialgias y compromiso variable del estado ge-

\footnotetext{
${ }^{1}$ Departamento de Epidemiología. División de Salud de las Personas, Ministerio de Salud.
} 
Epidemiología de la infección por hantavirus en Chile - V. Sotomayor P. y X. Aguilera S.

neral, seguido de compromiso pulmonar bilateral y síndrome de insuficiencia respiratoria del adulto, con una letalidad entre 50 y $75 \%$.

En Sudamérica, los primeros casos se produjeron entre 1993 y 1994 en Argentina y Brasil. En 1995, ocurrió un brote en la zona de El Bolsón, provincia de Río Negro, Argentina, identificándose un nuevo tipo de hantavirus llamado virus Andes ${ }^{7}$, cuyo reservorio es el ratón de cola larga (Oligoryzomys longicaudatus). En 1996, a raíz del estudio molecular y ecológico de otro brote ocurrido en Argentina, se planteó por primera vez la transmisión persona a persona ${ }^{8}$

En Paraguay se detectaron los primeros casos de SPH en 1995, causados por un nuevo tipo de hantavirus que recibió el nombre de virus Laguna Negra. Se caracteriza por ser menos agresivo, con una letalidad aproximada a $12 \%{ }^{9}$. En el resto de América se han reportado casos en Canadá (1994) y casos esporádicos en Uruguay, Bolivia y Panamá, donde recientemente se notificó un brote de $\mathrm{SPH}^{10}$.

Un estudio reciente demostró la diversidad genética entre los hantavirus de América del Sur existiendo, de acuerdo a su distribución geográfica, cinco linajes diferentes. Los casos de SPH de la Patagonia Argentina y Sur de Chile presentan un linaje común, denominado ADN Sur ${ }^{11}$.

\section{Antecedentes en Chile}

En Chile, el primer caso de SPH fue diagnosticado en octubre de 1995, en una mujer residente en la comuna de Cochamó, provincia de Llanquihue, X Región, quien sobrevivió a la enfermedad. Sin embargo, estudios serológicos han demostrado la presencia de anticuerpos contra hantavirus en personas que sobrevivieron a una neumonía atípica con gran distress respiratorio en 1993 y que pertenecían a 3 grupos familiares residentes en Niebla, Valdivia ${ }^{12}$.

Artículo al respecto en pág. 233

En cuanto al agente etiológico, en Chile sólo se ha identificado al virus Andes ${ }^{13}$, cuyo único reservorio comprobado hasta el momento es $O$. longicaudatus. Este roedor mostró una reactividad de $13 \%$ de anticuerpos contra el virus Sin Nombre en estudios ecológicos realizados en $1997 .{ }^{14}$

Al igual que en E.U.A, la manifestación clínica más relevante de la enfermedad por hantavirus es el SPH; sin embargo, también se han descrito casos de enfermedad leve (sin manifestaciones de compromiso pulmonar) y personas asintomáticas con evidencia serológica de infección por hantavirus ${ }^{15}$. Es así como estudios epidemiológicos realizados en Aysén, mostraron una prevalencia de infección por hantavirus que varió entre $2 \%$ en una zona urbana y $13,1 \%$ en un área endémica ${ }^{16}$. La prevalencia en el personal de salud fue similar a la observada en la comunidad $(3,8 \%)^{15}$. Estudios recientes realizados en trabajadores del Hospital de Temuco, demostraron que no existen evidencias serológicas de infección por hantavirus en personal expuesto a casos de SPH. ${ }^{16 a}$ Una encuesta serológica realizada en 130 residentes de Cochamó, provincia de Llanquihue, X Región, demostró evidencia serológica de infección por hantavirus en 6 personas sanas ${ }^{17}$.

Por otra parte, los casos chilenos han presentado una mayor frecuencia de signos hemorrágicos que los casos norteamericanos. En 1997 se publicó la primera descripción clínica de un caso de $\mathrm{SPH}$, correspondiente a un varón de 30 años, que falleció por síndrome de distress respiratorio. Esta persona presentó compromiso hemorrágico con hemotórax y edema pulmonar hemorrágico ${ }^{18}$. Asimismo, la caracterización clínica de los primeros 13 casos ocurridos en Aysén, mencionó la presencia de hematuria microscópica y cilindruria en algunos de ellos, así como la existencia de petequias en los niños ${ }^{19}$. En una revisión de 33 casos ocurridos en la Región de la Araucanía y Aysén, se observó que $60 \%$ de los casos presentaron petequias y trastornos hemorragíparos ${ }^{20}$.

\section{Sistema de Vigilancia}

En 1995, el Ministerio de Salud de Chile (MINSAL), frente a la situación ocurrida en la zona de El Bolsón, Argentina, elaboró las recomendaciones y estrategias de prevención y con- 
trol. Se preparó un documento técnico sobre las características de las infecciones por hantavirus y las fiebres hemorrágicas virales, con el objeto de alertar epidemiológicamente a los equipos de salud de los Servicios de Salud del país ${ }^{21}$.

A partir del primer caso confirmado de enfermedad por hantavirus, en 1995, se presentaron casos esporádicos hasta agosto de 1997, mes en que hubo un brote epidémico en la región de Aysén, con dos conglomerados de tipo familiar ${ }^{15}$. Frente a esta situación, el MINSAL convocó a un grupo de expertos nacionales e internacionales con el objeto de estudiar la epidemiología de la infección por hantavirus en Chile y establecer las primeras directrices y la normativa para un sistema de vigilancia epidemiológica. Se capacitó a los equipos de salud del sur del país y se efectúo transferencia tecnológica para la confirmación de los casos en el ISP de Chile, el que se constituyó como laboratorio de referencia nacional. Se iniciaron los primeros estudios ecológicos y la investigación de los conglomerados de casos ${ }^{22}$.

En febrero de 1998 se reformuló la normativa que establecía el sistema de vigilancia de la infección por hantavirus. Esta norma unificó los criterios de notificación e investigación epidemiológica, incluyendo la investigación de contactos y la normativa para la obtención, envío y transporte de muestras ${ }^{23}$.

Los objetivos del sistema de vigilancia de la infección por hantavirus son: detectar cambios inesperados en la distribución y ocurrencia del $\mathrm{SPH}$, evaluar su tendencia y patrón de presentación en el largo plazo, así como identificar cambios en el agente o factores del huésped. El sistema se establece sobre la base de tres componentes: clínico-epidemiológico, de laboratorio y ambiental. El Departamento. de Epidemiología del MINSAL, actúa como coordinador de la vigilancia epidemiológica.

A continuación se detallan los principales aspectos de esta vigilancia:

\section{Definición de caso}

La definición establecida en las normas de vigilancia que acompañan al decreto que regula la notificación de las Enfermedades de Declaración Obligatoria, es similar a la establecida por el CDC* e incluye el pródromo de síndrome gripal, considerando que en nuestro país esta enferme-

Cuadro 1. Definición de casos de síndrome pulmonar por hantavirus (SPH)

Caso sospechoso de SPH: Cuadro clínico caracterizado por una o más de las siguientes manifestaciones clínicas: cuadro febril $\left(\mathrm{T}^{\circ}\right.$ superior a $\left.38,3^{\circ} \mathrm{C}\right)$, que ocurre en una persona previamente sana, con un pródromo de síndrome gripal y que evoluciona con: distress respiratorio sin causa que lo explique, radiografía de tórax con edema intersticial difuso que semeja un síndrome de dificultad respiratoria aguda, compromiso respiratorio que requiere de oxigenación suplementaria, desarrollado dentro de las primeras 72 horas de hospitalización. Alternativamente, cuadro respiratorio inexplicable, con resultado de muerte y autopsia que demuestra edema pulmonar no cardiogénico, sin una causa identificable por laboratorio.

Caso confirmado de SPH: Caso clínicamente compatible con la definición de caso sospechoso, que ha sido confirmado por el Laboratorio de Referencia Nacional del ISP.

Criterios de laboratorio:

La confirmación del diagnóstico de SPH la realiza el Laboratorio de Referencia Nacional del ISP, mediante:

- Serología, detección de anticuerpos específicos IgM e IgG presentes en el suero o

- Transcripción reversa acoplada con reacción de polimerasa en cadena (TR-RPC), detección de material genético viral en sangre o tejidos o

- Inmunohistoquímica (IHQ), detección de antígenos virales en tejidos

Fuentes: Normas Técnicas Vigilancia Enfermedades Transmisibles. Ministerio de Salud de Chile. 2000. Referencia 23.

* MMWR. May 2, 1997, Vol 46, RR-10 p16. 
dad es endémica y el diagnóstico debe sospecharse en el período prodrómico. La definición de casos y criterios de laboratorio se presentan en el Cuadro 1.

Modalidad de vigilancia. En abril de 2000 se aprobó la modificación al decreto que regula la notificación de las Enfermedades de Declaración Obligatoria, estableciendo al SPH como de notificación inmediata frente a la sola sospecha de un caso $^{24}$. La normativa de este decreto incorpora además, la categoría de enfermedad leve por hantavirus, sin compromiso pulmonar y con evidencia serológica de infección aguda. Por otra parte, esta enfermedad es de notificación obligatoria para los países incorporados al MERCOSUR y a la Red de Vigilancia Epidemiológica del Convenio Hipólito Unanue.

La notificación del caso sospechoso de SPH se realiza a través del formulario único de notificación y envío de muestras. Es efectuada en forma inmediata desde el establecimiento que atiende el caso, a la Unidad de Epidemiología del Servicio de Salud, quien a su vez informa al Dpto. de Epidemiología del nivel central, que establece un registro único y centralizado de casos. Los casos sospechosos ingresan a una base de datos y, de acuerdo a los resultados de laboratorio, se confirman o descartan. Los resultados de esta vigilancia se difunden a través de la página web del Departamento de Epidemiología, http://www.epi.minsal.cl y se publican periódicamente en los boletines epidemiológicos del MINSAL. ${ }^{25,26}$

Manejo del caso y obtención de muestra. Frente a la sospecha clínica de un caso de SPH se indica su hospitalización inmediata en un centro que cuente con medios para enfrentar un distress respiratorio. Se obtiene una muestra de sangre, la que es enviada al Laboratorio de Referencia Nacional, para su confirmación o descarte. En caso de fallecer el paciente antes de obtener sangre por venopunción, se tomará mediante punción cardíaca.

Confirmación por el Instituto de Salud Pública. El ISP es responsable de confirmar los casos e informar el resultado en forma inmediata al médico tratante en el nivel local y a la Unidad de Epidemiología del Servicio de Salud de ocurrencia. La confirmación se realiza de acuerdo a los criterios descritos en el Cuadro 1.

Investigación epidemiológica. Frente a la no- tificación de un caso sospechoso, las Unidades de Epidemiología, en coordinación con los Departamentos de Programas del Ambiente, deben efectuar la investigación en terreno, cuyos objetivos son efectuar la evaluación epidemiológica de los casos y de personas en su entorno que pudieron estar expuestas a la infección, evaluando los factores de riesgo/exposición individual, a través de algún cuestionario y realizar la evaluación ecológica/ambiental, de acuerdo a la normativa establecida ${ }^{27}$. Se buscan las posibles fuentes de infección y se realiza educación referente a medidas de prevención y control de la enfermedad.

Seguimiento y obtención de muestras. Dependiendo de la evaluación de terreno y de la presencia de síntomas sugerentes de SPH, se puede indicar estudios serológicos en otras personas expuestas y someterlas a seguimiento.

Medidas de prevención y control. La información epidemiológica permite recomendar la aplicación de las medidas de prevención y control.

La educación sanitaria contribuye a mejorar la identificación y tratamiento de la enfermedad y disminuir el contacto de las personas con el reservorio. En este sentido, el MINSAL elaboró un documento técnico con orientaciones generales para el diagnóstico y manejo de los pacientes con $\mathrm{SPH}^{28}$ y preparó material educativo dirigido a los equipos de salud del sector público y privado ${ }^{29}$.

En 1998, el Ministerio de Salud realizó el II Seminario Internacional de Hantavirus, en coordinación con el Servicio de Salud Valdivia. Esta actividad tuvo como finalidad analizar y revisar la situación de esta enfermedad en el ámbito nacional e internacional, así como entregar y actualizar conocimientos.

En 1999, los Servicios de Salud Ñuble y Bío Bío, enfrentados al aumento de casos en la Región del Bío Bío, realizaron talleres locales dirigidos a los equipos de salud, con la participación de representantes de Epidemiología, Programas de Salud Ambiental y profesionales de los laboratorios y médicos clínicos.

Con el objeto de mejorar la identificación temprana de los casos de SPH, el Departamento de Epidemiología, en coordinación con Relaciones Públicas del MINSAL, elaboró material de difusión con el algoritmo recomendado por OPS, ${ }^{30}$ 
adaptado a la situación nacional. Este material, dirigido a los profesionales del sector salud, fue distribuido a todos los establecimientos de salud del país.

La información a la población general se ha canalizado a través de la Campaña de Prevención de Infección por Hantavirus, realizada por el MINSAL. Esta tiene por objeto promover la adopción de medidas de prevención en la población, además de informar sobre las características de los hantavirus y su mecanismo de transmisión. Entre 1997 y 1998 se realizaron campañas comunicacionales y de prensa y se distribuyó material educativo para la población general, profesores y turistas. ${ }^{31} \mathrm{En}$ los dos últimos años se han realizado campañas focalizadas en lugares donde se han presentado brotes de tipo familiar, como Bío Bío y Ñuble.

Los Servicios de Salud afectados por esta enfermedad realizan cada año campañas de prevención dirigidas principalmente a la población rural. En los meses estivales se incorporan medidas de prevención dirigidas a turistas y excursionistas.

Por otra parte, el Departamento de Epidemiología del MINSAL, incorporó en la página Web contenidos educativos dirigidos a la población general, en su sección Conozca más de hantavirus. ${ }^{32}$

\section{SITUACION EPIDEMIOLOGICA}

El SPH se presentó en forma de casos esporádicos hasta julio de 1997 y posteriormente en forma de brotes epidémicos en diferentes lugares del sur del país, para mantenerse actualmente como una endemia (Figura 1).

Entre 1993 y julio de 2000 se han confirmado 123 casos de SPH, incluyendo 5 casos estudiados en forma retrospectiva por el Servicio de Salud Valdivia y la Universidad Austral de Chile, Valdivia, X Región ${ }^{12}$ (Tabla 1).

Los casos se han concentrado en los meses más cálidos (noviembre a abril), con excepción de 1997, en que el mayor número de casos se presentó en agosto. Durante el año 2000 y hasta el mes de julio, se ha observado un mayor número de casos en los meses fríos, situación que no había ocurrido anteriormente. Este hecho podría explicarse por las condiciones climáticas durante este año, con un mayor volumen de precipitaciones en los meses de mayo y junio.

Características de los casos. Ha afectado preponderantemente a hombres $(75 \%)$, cuya edad promedio es de 29 años, con un rango de 2 a 75 años (Tabla 2). Los menores de 15 años representan el 15\% de los casos (18 casos), siendo mayor en los años 1997 y 1998, cuando ocurrió un brote en la Región de Aysén, que com-

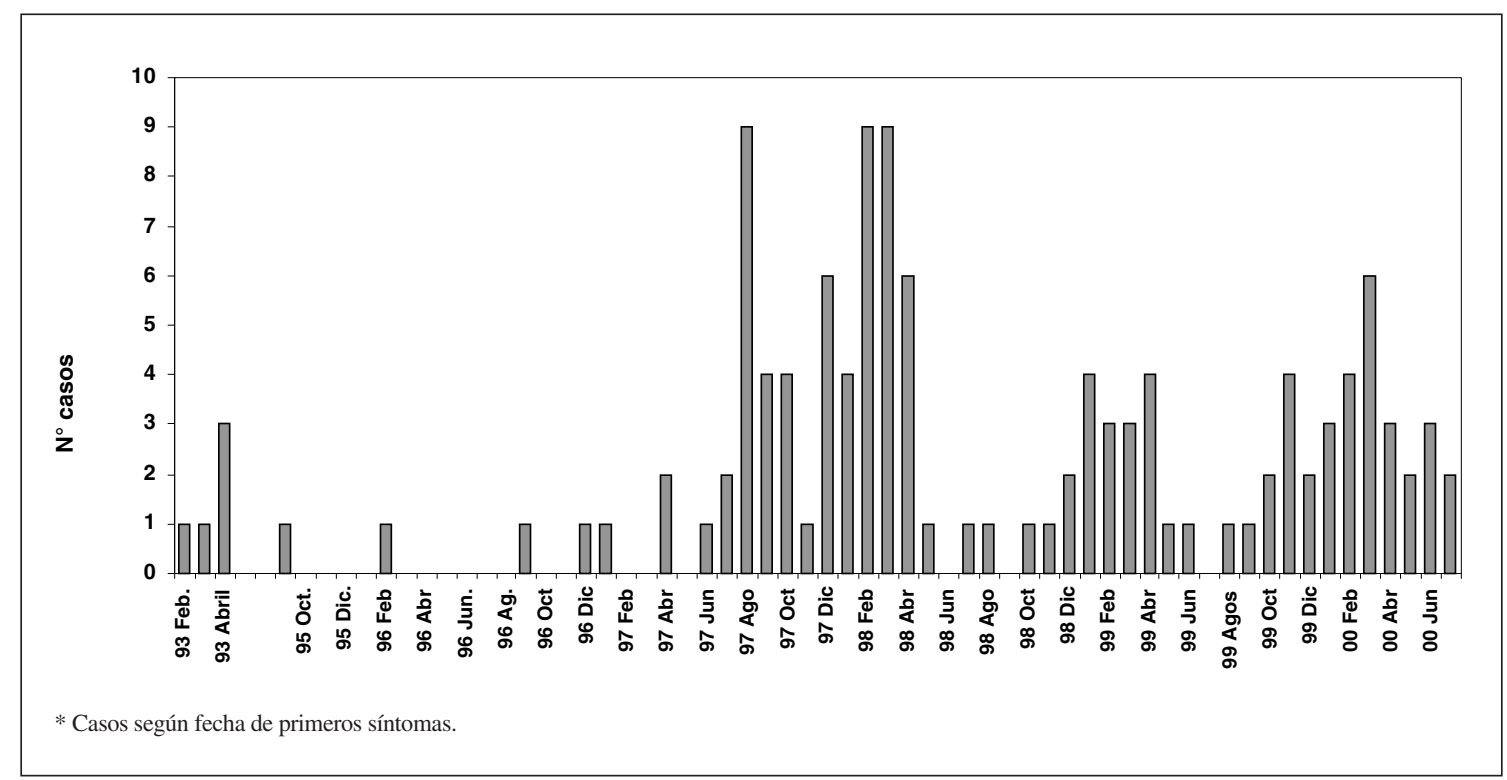

Figura 1. Casos de síndrome pulmonar por hantavirus, según mes de ocurrencia. Chile 1993-julio 2000. 
prometió a tres grupos familiares. El mayor riesgo de enfermar, de acuerdo a las tasas de incidencia por edad, se ha presentado en las edades productivas, especialmente entre los 20 a 39 años (Tabla 3).

Del total de casos acumulados de SPH, han fallecido 61 personas, lo que representa una letalidad global de $49,6 \%$, con una tendencia a la disminución en el transcurso de los últimos dos años (Tabla 2). En 1999 la letalidad llegó a $42 \%$, el valor más bajo respecto a los años anteriores. Esta disminución puede atribuirse en parte a la mejoría en la capacidad diagnóstica y al mayor conocimiento de la enfermedad que permite detectar y tratar a los casos en forma más oportuna. Un aspecto interesante de destacar ha sido la variación de la letalidad de acuerdo a la edad de los afectados, observándose una mayor

Tabla 1. Casos de síndrome pulmonar por hantavirus por Servicio de Salud de origen. Chile 1993 - Julio 2000

\begin{tabular}{|c|c|c|c|c|c|c|c|c|c|c|}
\hline \multirow[t]{2}{*}{ Región } & \multirow[t]{2}{*}{ Servicio } & \multirow[t]{2}{*}{ 1993* } & \multirow[t]{2}{*}{1995} & \multirow[t]{2}{*}{1996} & \multirow[t]{2}{*}{1997} & \multirow[t]{2}{*}{1998} & \multirow[t]{2}{*}{1999} & \multirow[t]{2}{*}{2000} & \multicolumn{2}{|c|}{$\begin{array}{c}\text { Acumulados } \\
1993-2000\end{array}$} \\
\hline & & & & & & & & & $\mathbf{n}$ & $\%$ \\
\hline VI & O'Higgins & 0 & 0 & 0 & 0 & 1 & 1 & 0 & 2 & 2 \\
\hline VII & Maule & 0 & 0 & 0 & 2 & 0 & 0 & 0 & 2 & 2 \\
\hline \multirow[t]{4}{*}{ VIII } & Ñuble & 0 & 0 & 0 & 1 & 3 & 5 & 4 & 13 & 11 \\
\hline & Bío-Bío & 0 & 0 & 0 & 1 & 2 & 2 & 3 & 8 & 7 \\
\hline & Concepción & 0 & 0 & 0 & 1 & 3 & 0 & 0 & 4 & 3 \\
\hline & Arauco & 0 & 0 & 0 & 0 & 0 & 1 & 1 & 2 & 2 \\
\hline \multirow[t]{2}{*}{ IX } & A.Norte & 0 & 0 & 0 & 1 & 4 & 1 & 5 & 11 & 9 \\
\hline & A.Sur & 0 & 0 & 0 & 2 & 8 & 5 & 4 & 19 & 15 \\
\hline \multirow[t]{3}{*}{$\mathrm{X}$} & Valdivia & 5 & 0 & 0 & 2 & 2 & 2 & 0 & 11 & 9 \\
\hline & Osorno & 0 & 0 & 0 & 0 & 1 & 5 & 2 & 8 & 7 \\
\hline & Llanchipal & 0 & 1 & 2 & 5 & 1 & 4 & 4 & 17 & 14 \\
\hline XI & Aysén & 0 & 0 & 1 & 15 & 10 & 0 & 0 & 26 & 21 \\
\hline Total & & 5 & 1 & 3 & 30 & 35 & 26 & 23 & 123 & 100 \\
\hline
\end{tabular}

Tabla 2.Casos de síndrome pulmonar por hantavirus. Características según año de ocurrencia. Chile 1993- Julio 2000

\begin{tabular}{|c|c|c|c|c|c|c|c|}
\hline Características & $1993 *$ & 1995 - 1996 & 1997 & 1998 & 1999 & $\begin{array}{c}2000 \\
\text { enero - julio }\end{array}$ & $\begin{array}{c}\text { Total } \\
\text { Casos } \\
\text { 1993-2000 }\end{array}$ \\
\hline $\mathrm{N}^{\circ}$ casos & 5 & 4 & 30 & 35 & 26 & 23 & 123 \\
\hline $\begin{array}{l}\text { Edad promedio } \\
\text { (años) }\end{array}$ & 27 & 25,8 & 32 & 26,7 & 28,9 & 30,7 & 29 \\
\hline Rango edad (años) & $3-59$ & $22-30$ & $2-60$ & $4-75$ & $8-52$ & $9-64$ & $2-75$ \\
\hline $\begin{array}{l}\mathrm{N}^{\circ}<15 \text { años } \\
(\%)\end{array}$ & 1 & 0 & $\begin{array}{c}5 \\
(16,7)\end{array}$ & $\begin{array}{c}6 \\
(17,1)\end{array}$ & $\begin{array}{c}3 \\
(11,5)\end{array}$ & $\begin{array}{c}3 \\
(13,0)\end{array}$ & $\begin{array}{c}18 \\
(14,6)\end{array}$ \\
\hline $\begin{array}{l}\mathrm{N}^{\circ} \text { hombres } \\
(\%)\end{array}$ & $\begin{array}{l}2 \\
-\end{array}$ & $\begin{array}{l}3 \\
-\end{array}$ & $\begin{array}{c}24 \\
(80,0)\end{array}$ & $\begin{array}{c}25 \\
(71,4)\end{array}$ & $\begin{array}{c}19 \\
(73,1)\end{array}$ & $\begin{array}{c}19 \\
(82,6)\end{array}$ & $\begin{array}{c}92 \\
(74,8)\end{array}$ \\
\hline $\begin{array}{l}\mathrm{N}^{\circ} \text { fallecidos } \\
\text { Letalidad }(\%)\end{array}$ & $\begin{array}{l}0 \\
-\end{array}$ & $\begin{array}{l}2 \\
-\end{array}$ & $\begin{array}{c}18 \\
(60,0)\end{array}$ & $\begin{array}{c}20 \\
(57,1)\end{array}$ & $\begin{array}{c}11 \\
(42,3)\end{array}$ & $\begin{array}{c}10 \\
(43,5)\end{array}$ & $\begin{array}{c}61 \\
(49,6)\end{array}$ \\
\hline
\end{tabular}


Epidemiología de la infección por hantavirus en Chile - V. Sotomayor P. y X. Aguilera S.

\begin{tabular}{|c|c|c|}
\hline \multicolumn{3}{|c|}{$\begin{array}{l}\text { Tabla 3. Casos de síndrome pulmonar por } \\
\text { hantavirus, según grupos de edad. Chile } 1993 \text { - } \\
\text { Julio } 2000\end{array}$} \\
\hline $\begin{array}{l}\text { Grupo } \\
\text { de edad (años) }\end{array}$ & $\mathbf{n}$ & $\begin{array}{c}\text { Incidencia } \\
\text { acumulada } \\
\text { Tasas/100.000 hab. }\end{array}$ \\
\hline $0-4$ & 4 & 0,27 \\
\hline $5-9$ & 6 & 0,42 \\
\hline $10-14$ & 8 & 0,60 \\
\hline $15-19$ & 9 & 0,73 \\
\hline $20-24$ & 21 & 1,73 \\
\hline $25-29$ & 16 & 1,31 \\
\hline $30-34$ & 20 & 1,64 \\
\hline $35-39$ & 17 & 1,51 \\
\hline $40-44$ & 8 & 0,85 \\
\hline $45-49$ & 4 & 0,51 \\
\hline $50-54$ & 2 & 0,30 \\
\hline $55-59$ & 4 & 0,75 \\
\hline 60 y más & 4 & 0,40 \\
\hline Total $*$ & 123 & 0,84 \\
\hline
\end{tabular}

sobrevida en los niños bajo 10 años de edad (Figura 2).

Como un indicador de calidad de la atención, se analizó el promedio de días de latencia entre la fecha de primeros síntomas y la fecha de hospitalización. En 108 casos con información dis-
Tabla 4. Actividad desarrollada por pacientes con síndrome pulmonar por hantavirus. Chile 1993 - Julio 2000

\begin{tabular}{lrr}
\hline Actividad & $\mathbf{n}$ & $\mathbf{\%}$ \\
\hline Trabajador agrícola o forestal & 54 & 49,5 \\
Dueña de casa & 15 & 13,8 \\
Estudiante & 14 & 12,8 \\
Otros & 9 & 8,3 \\
Actividad pesquera & 8 & 7,3 \\
Preescolar & 5 & 4,6 \\
Comercio & 4 & 3,7 \\
& \multicolumn{3}{l}{} \\
Total * & 109 & 100,0 \\
& \\
* 14 casos sin información. Incluye 5 casos estudiados retros- \\
pectivamente.
\end{tabular}

ponible, se observó una latencia de 4,2 días entre la fecha de inicio de síntomas y la hospitalización. No se observó diferencia entre los fallecidos (4,4 días) y los sobrevivientes (4,1 días). En los casos de fallecidos por SPH, la defunción se produjo como promedio, a los 6 días de iniciados los síntomas.

Con respecto a los factores asociados a la ocurrencia de los casos, si bien la información disponible en los formularios de notificación enviados al MINSAL no permite identificar con exactitud los factores de riesgo, existen algunas

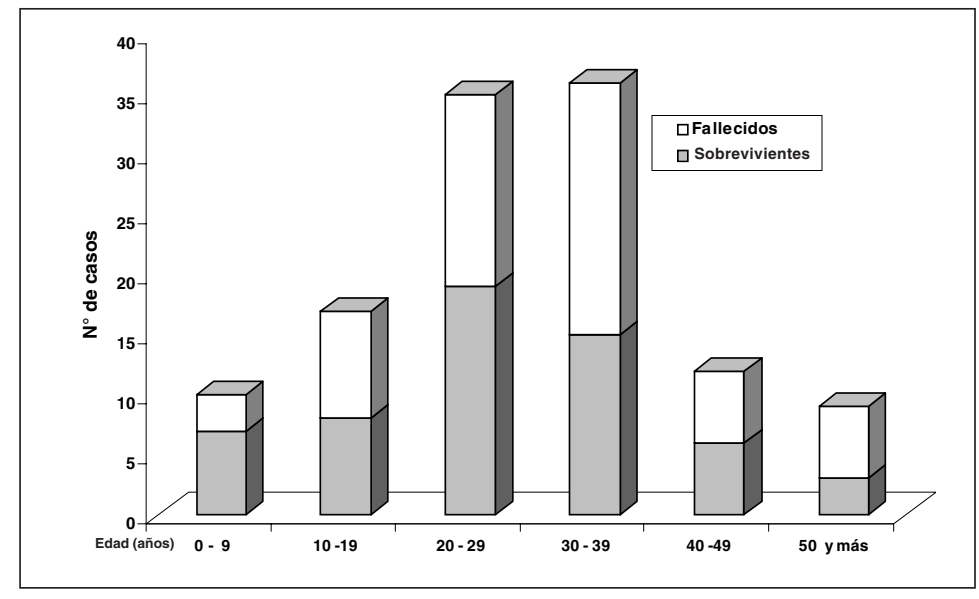

Figura 2. Casos de síndrome pulmonar por hantavirus según grupo de edad y resolución*. Chile 1995-julio 2000. * Excluye 5 casos estudiados en forma retrospectiva. 


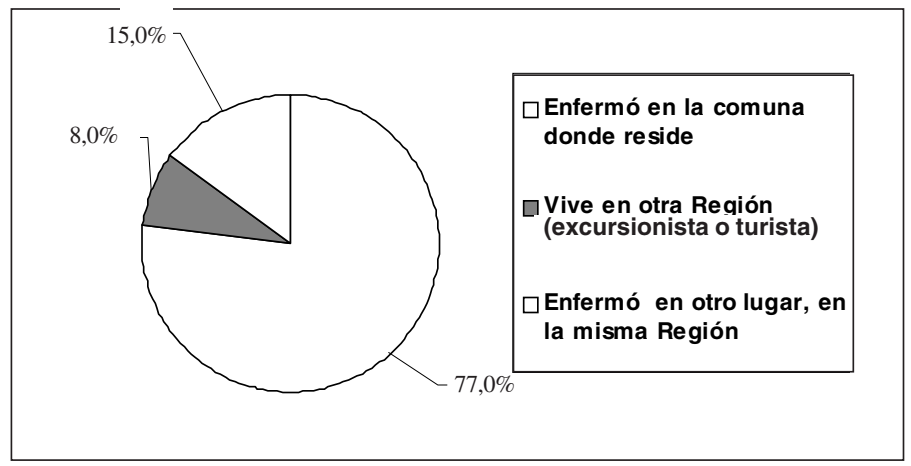

Figura 3. Casos de síndrome pulmonar por hantavirus, según residencia. Chile 1993-julio 2000.

características relevantes, como por ejemplo la ocupación, que puede estar asociada a un mayor riesgo de enfermar. De un total de 109 casos en que se obtuvo información sobre actividad ocupacional, $49,5 \%$, correspondían a trabajadores agrícolas o forestales, $14 \%$ realizaban actividades relacionadas con el hogar, $13 \%$ eran estudiantes y $7 \%$ realizaban actividades relacionadas con la pesca (Tabla 4). De acuerdo al lugar probable de infección, 77\% (94 casos) adquirieron la enfermedad en su lugar de residencia habitual, $15 \%$ (16 casos) la adquirió en otro lugar, dentro de la misma región de residencia y solamente $8 \%$ (10 casos) eran visitantes o turistas en la zona de probable infección (Figura 3).

En cuanto a la distribución geográfica, los casos de SPH se han presentado desde la VI hasta la XI Región, esto abarca una superficie de $291.484 \mathrm{~km}^{2}$, que representa el $38,5 \%$ de la superficie de Chile continental. La Región de Los Lagos (X) concentra el mayor número de casos, seguido de la Araucanía (IX) y Bío Bío (VIII), (Figura 4, Tabla 1).

A través del tiempo se ha observado un desplazamiento del riesgo de enfermar, comenzando por el extremo sur en dirección al norte (Figura 5). Es así como durante 1997 y 1998 las tasas más altas se presentaron en Aysén (16,5 y 10,8 por 100.000 hbtes. respectivamente), no volviendo a presentarse casos desde esa fecha. En 1998, en cambio, la Región de la Araucanía alcanzó el riesgo más elevado (1,4 por 100.000). En 1999, la Región de Los Lagos presentó el riesgo más alto (1,14 por 100.000$)$, superando a la Región de la Araucanía $(0,69$ por 100.000$)$ y Bío Bío (0,41 por 100.000). Hasta julio del 2000, el mayor número de casos se ha reportado en la Región de la Araucanía, con 9 casos (1,02 por 100.000), correspondiendo 5 de ellos a la provincia de Malleco, Servicio de Salud Araucanía Norte (2,28 por 100.000). El riesgo de esta población corresponde a uno de los más altos después de lo ocurrido en Aysén en 1997 (Figura 5).

Otro aspecto relevante es la distribución urbano-rural, observándose que el mayor porcentaje de casos se ha presentado en comunas con un alto nivel de ruralidad. En la Región de Aysén las comunas más afectadas son: Coyhaique (9 casos), Lago Verde (6 casos) y Chile Chico (4 casos); en la Región de Los Lagos: Valdivia

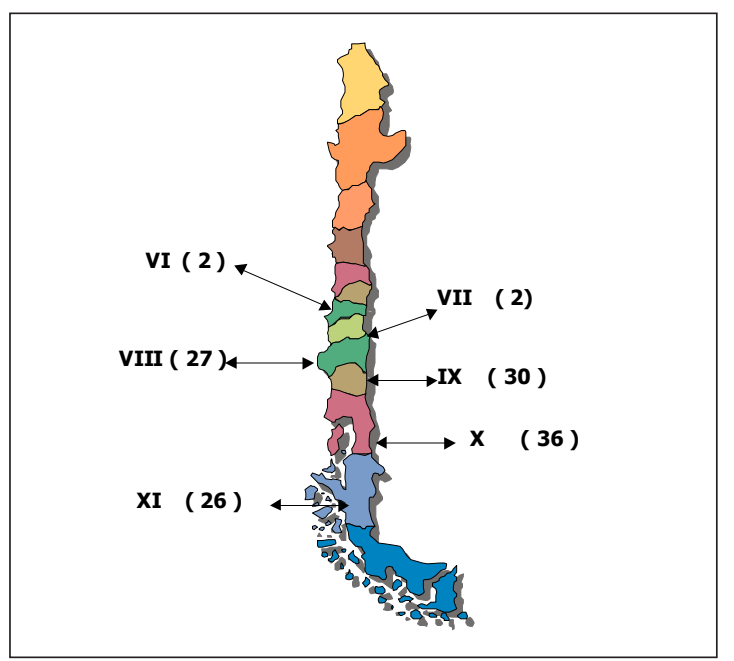

Figura 4. Casos confirmados de síndrome pulmonar por hantavirus según región. Chile 1993-julio 2000. 
(6 casos), Cochamó, y Purranque (4 casos cada uno); en la Región de la Araucanía: Cunco (6 casos), Curacautín (5 casos), Villarrica y Melipeuco ( 4 casos cada uno); en la Región del Bío Bío: El Carmen (5 casos), Mulchén, Los Angeles y Tucapel (3 casos cada uno).

Las características epidemiológicas de los casos son similares en las distintas regiones del país. Sin embargo, en la Araucanía, la edad promedio de los casos es discretamente inferior, en comparación con Bío Bío y Aysén. Por otra parte, en la Región del Bío Bío, la proporción de mujeres es mayor, en relación a lo observado en otras regiones (Tabla 5).

La letalidad más baja se observó en Aysén $(42,3 \%)$, la que puede ser atribuida a la experiencia en el manejo de los casos y al uso de medidas terapéuticas específicas. La mayor letalidad se presenta en la Región del Bío Bío $(63,0 \%)$, donde ocurrieron dos brotes familiares: El Carmen, en el Servicio de Salud Ñuble y Mulchén, en el Servicio de Salud Bío Bío.

Finalmente, cabe mencionar que entre los años 1995 y 2000, se han detectado 10 casos con enfermedad leve por hantavirus, los que evolucio- naron sin mayor compromiso pulmonar. Esto representa 7,5\% del total de casos confirmados (123 casos de SPH con 10 casos de enfermedad leve). Cinco de 10 casos con enfermedad leve por hantavirus han ocurrido entre los conglomerados. La información disponible no permite analizar en detalle las características clínicas de estos casos, ya que la mayoría corresponde a hallazgos realizados en la investigación epidemiológica y serológica de casos de SPH.

Conglomerados de casos. Esta enfermedad se ha caracterizado por presentarse en forma de casos aislados y esporádicos, junto con la ocurrencia de casos relacionados en el tiempo y espacio (clusters). A continuación se presentan las características de estos conglomerados.

Entre agosto de 1997 y principios de 1998, se presentaron en la Región de Aysén, casos de SPH correspondientes a tres grupos familiares. Los estudios epidemiológico, ecológico y molecular realizados en este brote, sugieren una estrecha relación entre los casos de uno de estos conglomerados. En opinión de los autores, no pudo excluirse la transmisión persona - perso$\mathrm{na}^{15}$.

Tabla 5. Casos de síndrome pulmonar por hantavirus. Variables biomédicas según Región y Servicio de Salud de ocurrencia. Chile 1993- Julio 2000

\begin{tabular}{|c|c|c|c|c|c|c|c|c|}
\hline \multirow{3}{*}{ Regic } & \multirow{3}{*}{$\begin{array}{c}\text { Servicio } \\
\text { de } \\
\text { Salud }\end{array}$} & \multirow[b]{3}{*}{$\mathbf{n}$} & \multicolumn{4}{|c|}{ Variables biomédicas } & \multirow{2}{*}{\multicolumn{2}{|c|}{ Letalidad }} \\
\hline & & & \multirow{2}{*}{\multicolumn{2}{|c|}{$\begin{array}{c}\text { Edad (años) } \\
\text { Promedio (Rango) }\end{array}$}} & \multicolumn{2}{|c|}{ Hombres } & & \\
\hline & & & & & n & $\%$ & \multirow{2}{*}{$\frac{\mathbf{n}}{2}$} & \multirow{2}{*}{$\%$} \\
\hline VI & O Higgins & 2 & 30 & $(25-36)$ & 2 & 100,0 & & \\
\hline VII & Maule & 2 & 43 & $(30-56)$ & 1 & 50,0 & 2 & - \\
\hline \multirow[t]{5}{*}{ VII } & Ñuble & 13 & & & & & 9 & \\
\hline & BíoBío & 8 & & & & & 7 & \\
\hline & Concepción & 4 & & & & & 1 & \\
\hline & Arauco & 2 & & & & & 0 & \\
\hline & Total regional & 27 & 29 & $(4-75)$ & 18 & 66,7 & 17 & 63,0 \\
\hline \multirow[t]{3}{*}{ IX } & Araucanía Norte & 11 & & & & & 6 & \\
\hline & Araucanía Sur & 19 & & & & & 9 & \\
\hline & Total regional & 30 & 26 & $(2-57)$ & 24 & 80,0 & 15 & $\mathbf{5 0 , 0}$ \\
\hline \multirow[t]{4}{*}{$\mathrm{X}$} & Valdivia* & 6 & & & & & 2 & \\
\hline & Osorno & 8 & & & & & 3 & \\
\hline & LLanchipal & 17 & & & & & 9 & \\
\hline & Total regional & 31 & 29 & $(5-63)$ & 24 & 77,4 & 14 & 45,1 \\
\hline \multirow[t]{2}{*}{ XI } & Aysén & 26 & 32 & $(2-60)$ & 21 & 80,8 & 11 & 42,3 \\
\hline & Total nacional* & 118 & 29,2 & $(2-75)$ & 90 & 76,2 & 61 & 51,6 \\
\hline
\end{tabular}




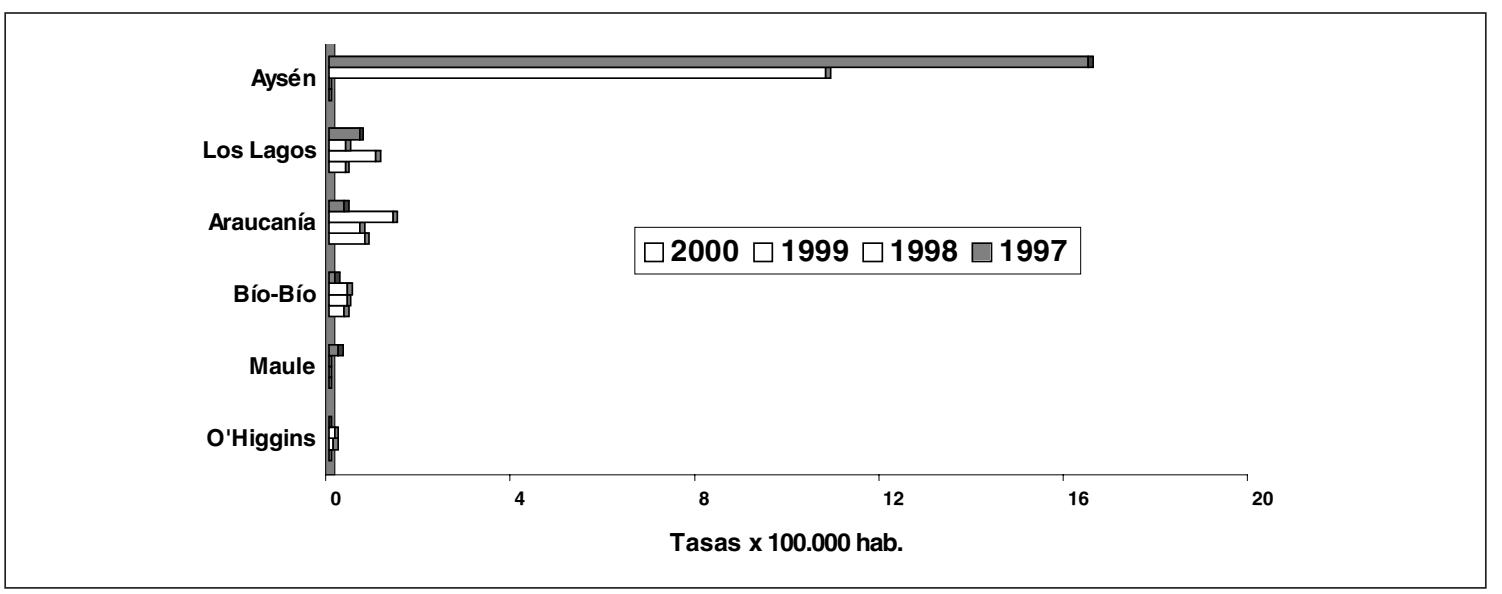

Figura 5. Tasas de incidencia de síndrome pulmonar por hantavirus, según lugar de ocurrencia. Chile 1997-julio 2000 .

Entre diciembre de 1997 y abril de 1998 se observó un brote en la Región de la Araucanía, confirmándose 13 casos de SPH. Anteriormente se había confirmado sólo un caso en el mes de agosto. También se presentaron casos en tres conglomerados, dos de ellos en la Araucanía. El primero ocurrió en Melipeuco y afectó a dos personas. Un niño de 2 años que sobrevivió y un familiar cercano, que enfermó 28 días después falleciendo a los seis días de evolución. Ambos participaron en una actividad doméstica en el hogar del caso índice.

El segundo grupo de casos ocurrió en Villarrica y correspondió a una persona que realizaba actividades agrícolas y su hijo de 5 años. El caso índice enfermó el 11 de febrero y a los 24 días enfermó el hijo. El pequeño acompañaba al padre a sus labores agrícolas. Ambos fallecieron.

Un tercer grupo de casos se presentó en Osorno, X Región, en una mujer que enfermó dos días después que su conviviente, este último presentó un cuadro de enfermedad leve por hantavirus, sin compromiso pulmonar. Una tercera persona asintomática, que vivía con la mujer, presentó evidencia serológica de infección por hantavirus.

Durante 1999, se presentó el menor número de casos de los últimos tres años; sin embargo, se presentaron 4 brotes de casos relacionados entre sí, dos de ellos en la VIII Región. El primero, ocurrió en El Carmen, provincia de Nuble y afectó a 3 personas: la madre, el yerno que enfermó a los dos días (ambos fallecieron) y la hija del caso índice. Esta última enfermó a los 9 días del primer caso y evolucionó con un cuadro de enfermedad leve por hantavirus, sin compromiso pulmonar. El segundo conglomerado se presentó en Mulchén, provincia de Bío Bío y afectó a 3 personas: una mujer de 52 años, su nieto que enfermó a los 21 días y un tío abuelo del niño. De este último no se obtuvo muestra para confirmación, falleció por un cuadro de distress respiratorio, correspondiendo probablemente al caso índice. El menor fue confirmado por la muestra de sangre tomada durante la investigación epidemiológica, cuando no presentaba síntomas. Posteriormente, se hospitalizó y falleció. Los dos casos confirmados participaron en la limpieza de la casa del tío abuelo del niño.

El tercer grupo de casos se presentó en tres trabajadores de San Pedro, Osorno, X Región, quienes compartían una cabaña y realizaban labores de pesca artesanal. El primero se enfermó el 27 de octubre, al tercer y cuarto día se enfermaron las otras 2 personas, falleciendo uno de ellos. Posteriormente el estudio serológico de los contactos, evidenció una infección por hantavirus en una mujer asintomática que cuidaba y visitaba la casa.

El último grupo afectó a una familia, residente de Los Muermos, provincia de Llanquihue, X Región. Este brote se caracterizó por presen- 
tar casos con una manifestación más benigna. El caso índice enfermó el 5 de noviembre, la madre presentó síntomas a los 5 días. Ambos evolucionaron con un cuadro pulmonar de menor gravedad, siendo clasificados como SPH. El tercer caso correspondió al padre, quien evolucionó con un cuadro de enfermedad leve por hantavirus, sin compromiso pulmonar y no requirió hospitalización.

\section{COMENTARIOS}

La vigilancia de esta enfermedad ha permitido monitorear su evolución en el tiempo. El SPH se ha comportado como casos esporádicos, llegando actualmente a ser una enfermedad endémica en nuestro país, con la característica de presentarse en brotes de tipo familiar o conglomerados de casos.

Se prevé que se mantendrá como una endemia de presentación estacional, similar a otros países, concentrándose los casos en los meses de primavera y verano. La variación del número de casos está relacionada a las condiciones climáticas, por lo que no se puede excluir un aumento de casos en el próximo período, por sobre lo observado en 1999.

El análisis descriptivo de los casos permite establecer las características epidemiológicas de las personas afectadas. Esta enfermedad afecta principalmente a hombres adultos jóvenes, residentes en sectores rurales y se asocia a actividades agrícolas-forestales o domésticas en zona rural. Es escasa la ocurrencia de casos entre personas que realizan actividades recreativas o turísticas. Esta información ha permitido que los equipos de salud locales, responsables de la prevención y el control, fortalezcan las actividades educativas dirigidas a la población general y rural, especialmente en aquellos lugares donde se han presentado situaciones de riesgo epidemiológico.

El alto porcentaje de casos en personas bajo 15 años de edad es una característica muy particular de los casos chilenos. En E.U.A. se observa una menor proporción en este grupo de edad $(5 \%)^{3}$, lo que podría ser explicado por la mayor participación en Chile de los niños en actividades relacionadas con tareas domésticas o agrícolas.
La letalidad ha bajado de $60 \%$ en 1997 , a $43 \%$ en el año 2000, esta disminución también se ha observado en otros países ${ }^{3}$ y puede atribuirse a la mejor capacidad diagnóstica de los equipos médicos y a la atención más oportuna de los casos. Sin embargo, existen diferencias entre los distintos Servicios de Salud, que pueden estar reflejando desigualdades en el acceso a la atención médica.

Los factores de riesgo asociados a esta enfermedad se han expresado a través de la actividad ocupacional de los casos, por lo que deben orientarse las medidas de prevención y control a los grupos de población más expuestos. Sin embargo, la realización de estudios de casos y controles, que han demostrado ser útiles para identificar los factores de riesgo y/o exposición, permitirían conocer las diferencias entre los casos y los no casos. Este aspecto es una tarea a futuro que deberá ser abordado con la colaboración de los investigadores y médicos clínicos.

Esta enfermedad se ha desplazado desde el extremo sur, hacia el norte del país, extendiéndose desde la VI a la XI Región, lo que corresponde a casi toda el área de distribución del reservorio.

Los riesgos han variado en el tiempo. Al comienzo (años 1997 y 1998) el mayor riesgo se concentró en la Región de Aysén, que desde 1999 no ha notificado casos. Este silencio epidemiológico puede ser atribuido a cambios en la población de roedores en esa zona.

Actualmente, el riesgo de enfermar por hantavirus se ha concentrado en la IX Región y especialmente en la provincia de Malleco.

La existencia de casos con sintomatología leve, sin compromiso pulmonar, y la presentación clínica más benigna en algunos casos con $\mathrm{SPH}$, determina la presencia de una enfermedad con variaciones en su expresión clínica. La inclusión de la enfermedad leve al sistema de vigilancia permitirá conocer sus características y real magnitud.

En el sistema de vigilancia, la retroalimentación y diseminación de la información a los niveles locales en forma permanente, cumple una función fundamental, siendo responsabilidad de los equipos de epidemiología en los distintos niveles de atención. Los médicos clínicos y personal de salud juegan un papel importante en la 
Epidemiología de la infección por hantavirus en Chile - V. Sotomayor P. y X. Aguilera S.

identificación temprana de los casos de enfermedad por hantavirus y por lo tanto la aplicación de las medidas de prevención y control.

La vigilancia debe tener un enfoque integrado, considerando los aspectos clínicoepidemiológicos, de laboratorio y ambientales. Por lo tanto la aparición de un caso debe alertar no sólo a los epidemiólogos, sino también a los médicos clínicos, personal de laboratorio y encargados de Programas de Control Ambiental, los que deben en conjunto enfrentar el aumento excesivo de casos en una comunidad o la aparición de un caso en una zona donde no existía con anterioridad. Frente a estas situaciones, deben buscarse los factores de exposición o riesgo, especialmente si surgen conglomerados. Los casos relacionados ofrecen una buena posibilidad de estudiar la enfermedad. Actualmente no se ha completado el estudio de los conglomerados ocurridos en estos últimos años. La caracterización y estudios moleculares de estos casos, como también los estudios de reservorios, contribuirán al conocimiento de esta enfermedad y permitirán abordar los mecanismos de transmisión. Este aspecto debe ser realizado con el apoyo de investigadores y recursos especialmente destinados para ello.

Los epidemiólogos debemos contribuir al conocimiento de esta enfermedad e impulsar el estudio de estos brotes, además de reforzar la capacidad de respuesta local a pesar de las limitaciones de recursos humanos y de apoyo logístico.

\section{RESUMEN}

El síndrome pulmonar por hantavirus (SPH) es una enfermedad emergente en Chile, 123 casos de SPH se confirmaron entre 1993 y julio de 2000, incluyendo 5 casos estudiados retrospectivamente. Esta enfermedad ha afectado especialmente a personas jóvenes (26 años promedio edad), en su mayoría hombres (75\%). Los casos se han presentado en personas residentes de comunidades rurales que realizaban actividades agrícolas-forestales o actividades relacionadas con el hogar. La letalidad global ha sido de 49,6\%, variando desde $60 \%$ en 1997 a $43 \%$ en el año 2000. Los casos se han presen- tado desde la VI a XI regiones del país, los riesgos más altos han ocurrido en Aysén (19971998) y Araucanía (1998 y 2000), destacando la ocurrencia de conglomerados de casos, en las Regiones de la Araucanía, Bío Bío, Los Lagos y Aysén. El SPH es una enfermedad de presentación endémica con aumento de casos en los meses de primavera y verano.

\section{BIBLIOGRAFIA}

1.- MORSE STEPHEN S. Factors in the emergence of infectious diseases. Emerg Infect Dis 1995; 1(1): 715.

2.- SCHMALJOHN C, HJELLE B. Hantaviruses: A global diseases problem. Emerg Infect Dis 1997; 3(2): 95-104.

3.- ORGANIZACION PANAMERICANA DE LA SALUD. Cuaderno Técnico 47; Hantavirus en las Américas: Guía para el diagnóstico, el tratamiento, la prevención y el control. Organización Panamericana de la Salud. Washington, D.C., 1999; pp 6-10.

4.- DUCHIN J S, KOSTER F T, PETERS C J et al. Hantavirus pulmonary syndrome: A clinical description of 17 patients with a newly recognized disease. N Engl J Med 1994; 330: 945-9.

5.- KHAN A S, KSIAZECK T G, PETERS C J. Hantavirus pulmonar syndrome. Lancet 1996; 347: 739-41.

6.- NICHOL S, SIPOROLOUS C, MORZUNOV S et al. Genetic identification of a hantavirus associated with an outbreack of acute respiratory illness. Science 1993; 262: 914-7.

7.- LOPEZ N, PADULA P, ROSSI C et al. Genetic identification of a new hantavirus causing severe pulmonary syndrome in Argentina. Virology 1996; 220: 223-6.

8.- PADULA P J, EDELSTEIN A, MIGUEL S D et al. Hantavirus pulmonary syndrome outbreak in Argentina: molecular evidence for person to person transmission of Andes virus. Virology 1998; 241: 323-30.

9.- WILLIAMS R J, BRYAN J N, MILLS R et al. An outbreak of hantavirus pulmonary syndrome in western Paraguay. Am J Med Hyg 1997; 57: $274-$ 82.

10.- Anónimo. MMWR, 17 marzo 2000, 49 (10): 205-7.

11.- PADULA P J, COLAVECCHIA S B, MARTINEZ $\mathrm{V} P$ et al. Genetic diversity, distribution, and serological features of hantavirus infection in five countries in South America. J Clin Microbiol 2000; 38 (8): 3029-35

12- NAVARRETE M, MANSILLA R, SALDIAS F, FERRES M, ZAROR L. Boletín Epidemiológico EpiVisión 1998; 19 (1): 3.

13.- LOPEZ N, PADULA P, ROSSI $\mathrm{C}$ et al. Genetic 
characterization and phylogeny of Andes virus and variants from Argentina and Chile. Virus Res 1997; 50: 77-84.

14.- PAVLETIC C, ELLIS B, MURUA R et al. An outbreak of infection by hantavirus pulmonar syndrome related to a rodent eruption in southern Chile. The Fourth International Conference HFRS and Hantavirus, 5-7 de Marzo de 1998, Atlanta, Georgia, 115.

15.- TORO J, VEGA J, MILLS J, KHAN A et al. An outbreak of hantavirus pulmonary syndrome, Chile 1997. Emerg Infect Dis 1998; 4: 684-7.

16.- VALDERRAMA R, VEGA J, TERRY W et al. Community serological survey of infection by hantavirus in the XI Region, Aysén, Chile. The Fourth International Conference HFRS and Hantavirus, 5-7 de Marzo de 1998, Atlanta, Georgia, 155.

16a.- CASTILLO C, MARDONES J, VILLAGRA E. Prevalencia de anticuerpos por antihantavirus en personal de salud en contacto con pacientes portadores de síndrome pulmonar por hantavirus. Temuco 1997 a 1999. Rev Méd Chile 2000; 128: 735-9.

17.- Ministerio de Salud de Chile. Hanta: La Respuesta Chilena. MINSAL, 1998 - Noviembre, pp 41-3.

18.- TAPIA M. Caso Clínico: Síndrome pulmonar por Hantavirus. Rev Chil Enf Respir 1997 13: 103-10.

19.- TAPIA M, MANSILLA C, VILLALON H, VALLEJOS B, VERA J L, GALLEGOS N et al. Hantavirus Pulmonary Syndrome. Clinical description of thirteen cases in the XI Region, Aysén, Chile. The Fourth International Conference HFRS and Hantavirus, 57 de Marzo de 1998, Atlanta, Georgia, 36.

20.- TAPIA M. Diagnóstico Clínico. Informe "Segundo Seminario Internacional de Hantavirus. Ministerio de Salud de Chile. Valdivia, 18-20 de Noviembre de 1998.

21.- MINISTERIO DE SALUD DE CHILE. Informe Técnico $N^{\circ}$ 13, Mayo 1995. Fiebres Hemorrágicas Virales: Hantaan, Ebola, Lassa.
22.- Informe final de las actividades realizadas por la Comisión Conjunta - Centers for Disease Control and Prevention de Estados Unidos de América, Ministerio de Salud de Chile, Organización Panamericana de la Salud, y ANLIS, Argentina- en relación a la infección por Hantavirus en Chile. Rev Chil Infect 1997; 14 (2): 123-34.

23.- MINISTERIO DE SALUD DE CHILE, CIRCULAR 4F/09. Infección por Hantavirus: Vigilancia Epidemiológica y Diagnóstico de Laboratorio, 9 de febrero 1998.

24.- Decreto 712, Reglamento sobre Enfermedades de Declaración Obligatoria. Diario Oficial 12 de abril de 2000. Ministerio de Salud de Chile

25.- AGUILERA X. Situación Epidemiológica del Síndrome Pulmonar por Hantavirus. Boletín Epidemiológico EpiVisión 1998; 19 (1): 2-3.

26.- SOTOMAYOR V. Síndrome Pulmonar por Hantavirus. Boletín de Vigilancia Epidemiológica El Vigía 1998; 1 (5): 2-3.

27.- MINISTERIO DE SALUD DE CHILE, CIRCULAR 9B/ $\mathrm{N}^{\circ} 52$. Intervención Ambiental en caso de focos de virus Hanta, 1 de diciembre de 1997.

28.- MINISTERIO DE SALUD, ORD.N /5136. Documento "Infección por Hantavirus: Diagnóstico y Tratamiento. Subcomisión de Investigación Clínica: Grupo de Trabajo Asesor Enfermedad por Hantavirus. Octubre 1997.

29.- Control y Prevención de la Infección por Hantavirus. Cuadernillo para el equipo del sector público de los Servicios de Salud. Ministerio de Salud. Noviembre 1997.

30.- ORGANIZACION PANAMERICANA DE LA SALUD. Cuaderno Técnico $N^{\circ}$ 47: Hantavirus en las Américas. Guía para el diagnóstico, el tratamiento, la prevención y el control. Organización Panamericana de la Salud, Washington DC, 1999: pp 17-22.

31.- Hanta: La Respuesta Chilena. Ministerio de Salud de Chile. Noviembre 1998.

32.- http://www.epi.minsal.cl

Correspondencia a:

E.U. Viviana Sotomayor Proschle

Fax (56-2) 630.0505

e-mail: vsotomay@minsal.cl 